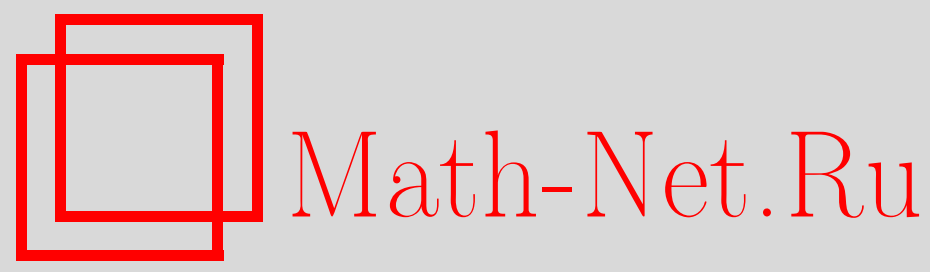

Е. Е. Дьяконова, Многотипные ветвящиеся процессы, эволюционирующие в марковской среде, Дискрет. матем., 2012, том 24, выпуск 3, 130-151

DOI: https://doi.org/10.4213/dm1204

Использование Общероссийского математического портала Math-Net.Ru подразумевает, что вы прочитали и согласны с пользовательским соглашением http://www . mathnet.ru/rus/agreement

Параметры загрузки:

IP : 3.81 .55 .215

26 апреля 2023 г., $11: 14: 52$ 
УДК 519.2

\title{
Многотипные ветвящиеся процессы, эволюционирующие в марковской среде
}

\author{
() 2012 г. Е. Е. Дьяконова
}

\begin{abstract}
Рассматриваются многотипные ветвящиеся процессы в случайной марковской среде, обладающие свойством критичности. Получена условная предельная теорема о распределении числа частиц в процессе в момент времени $n$ при условии невырождения процесса к этому моменту.

Работа выполнена при поддержке Российского фонда фундаментальных исследований, проект 11-01-00139.
\end{abstract}

\section{1. Введение и основные результаты}

Впервые ветвящийся процесс в случайной среде был рассмотрен Смитом и Вилкинсоном [1]. В их работе среда, в которой эволюционирует процесс, порождалась последовательностью независимых одинаково распределенных случайных величин. Вскоре Атрейя и Карлин [2] исследовали свойства ветвящихся процессов, функционирующих в случайной среде более общего вида. С тех пор появилось много работ, в которых изучаются ветвящиеся процессы в случайной среде (см., например, [3-23]). Так, в [3, 5] для многотипных ветвящихся процессов в случайной среде были найдены условия невырождения с вероятностью единица и установлены предельные теоремы о распределении числа частиц в процессе. Для среды, порожденной последовательностью независимых одинаково распределенных случайных величин, многотипный докритический ветвящийся процесс с финальным продуктом был исследован в [14], а докритический многотипный ветвящийся процесс с иммиграцией был изучен в $[15,22]$. Вероятность невырождения критических и докритических однотипных ветвящихся процессов для случая стационарной случайной среды анализировалась в [21].

До появления статей [7, 10], стандартным условием, накладываемым на критический ветвящийся процесс, эволюционирующий в случайной среде, было предположение о конечности второго момента $\mathbf{E} X^{2}<\infty$ приращения $X$ сопровождающего случайного блуждания. Именно при этом условии (и соответственно, при его многомерном аналоге в многотипном случае) в случае среды, порожденной последовательностью независимых одинаково распределенных случайных величин, в [23,9] была исследована асимптотика вероятности невырождения для критических однотипных ветвящихся процессов, а для критических многотипных ветвящихся процессов - в [17]. В последующих работах [7, 10-12, 20] была получена асимптотика вероятности невырождения и изучено поведение числа частиц в процессе для гораздо более богатого класса критических ветвящихся процессов с одним типом частиц, функционирующих в случайной среде, порожденной последовательностью независимых одинаково распределенных случайных величин. Для 
представителей этого класса не только конечность второго момента $\mathbf{E} X^{2}$ не обязательна, но даже и существование математического ожидания у случайной величины $X$ не является необходимым требованием. Многотипные ветвящиеся процессы в случайной среде, порожденной последовательностью независимых одинаково распределенных случайных величин, рассматривались в $[16,18]$ в предположении, что сопровождающие случайные блуждания этих процессов удовлетворяют условию Спицера-Дони (см. ниже условие С1), а матрицы средних значений законов размножения частиц всех поколений имеют один и тот же общий левый (или правый) собственный вектор, соответствующий их перроновым корням.

В [19] исследовалась асимптотика вероятности невырождения многотипных ветвящихся процессов в случайной марковской среде, обладающих свойством критичности. В настоящей работе для этого класса ветвящихся процессов изучается предельное поведение числа частиц в процессе в момент времени $n \rightarrow \infty$. В частности, мы обобщаем некоторые результаты [7, 16, 19].

Для описания интересующей нас модели ветвящегося процесса в случайной среде потребуется ряд стандартных обозначений. Пусть $\mathbf{e}_{j}, j=1, \ldots, p,-p$-мерная векторстрока, $j$-я компонента которой равняется 1, а все остальные компоненты - нули, $\mathbf{0}=(0, \ldots, 0)-p$-мерная нулевая вектор-строка, а $\mathbf{1}=(1, \ldots, 1)-p$-мерная единичная вектор-строка. Для

$$
\mathbf{x}=\left(x_{1}, \ldots, x_{p}\right), \quad \mathbf{y}=\left(y_{1}, \ldots, y_{p}\right)^{\prime}
$$

положим

$$
|\mathbf{x}|=\sum_{i=1}^{p}\left|x_{i}\right|, \quad(\mathbf{x}, \mathbf{y})=\sum_{i=1}^{p} x_{i} y_{i}
$$

будем обозначать $\mathbf{x} \otimes \mathbf{y}$ матрицу размера $p \times p$, имеющую элементы $x_{i} y_{j}, 1 \leqslant i, j \leqslant p$; здесь и далее штрих обозначает транспонирование. Для положительной матрицы $A=(A(i, j))_{i, j=1}^{p}$ обозначим $\rho(A)$ ее перронов корень и введем норму

$$
\|A\|=\sum_{i, j=1}^{p} A(i, j) .
$$

Пусть $\mathbf{v}(A)=\left(v_{1}(A), \ldots, v_{p}(A)\right)$ - левый, а $\mathbf{u}(A)=\left(u_{1}(A), \ldots, u_{p}(A)\right)^{\prime}-$ правый положительные собственные векторы матрицы $A$, соответствующие собственному значению $\rho(A)$ и удовлетворяющие условиям

$$
|\mathbf{u}(A)|=1, \quad(\mathbf{v}(A), \mathbf{u}(A))=1 .
$$

Пусть

$$
\begin{gathered}
\mathbf{J}^{p}=\left\{\mathbf{s}=\left(s_{1}, \ldots, s_{p}\right): 0 \leqslant s_{i} \leqslant 1, i=1, \ldots, p\right\} \\
\mathbf{N}_{0}=\{0,1,2, \ldots\}, \quad \mathbf{N}_{0}^{p}=\left\{\mathbf{t}=\left(t_{1}, \ldots, t_{p}\right): t_{i} \in \mathbf{N}_{0}, i=1, \ldots, p\right\} .
\end{gathered}
$$

Для $\mathbf{s}=\left(s_{1}, \ldots, s_{p}\right) \in \mathbf{J}^{p}$ и $\mathbf{t}=\left(t_{1}, \ldots, t_{p}\right) \in \mathbf{N}_{0}^{p}$ положим

$$
\mathbf{s}^{\mathbf{t}}=\prod_{i=1}^{p} s_{i}^{t_{i}}
$$


Пусть $\zeta=\left\{\zeta_{n}, n \in \mathbf{N}_{0}\right\}$ - неприводимая положительно возвратная цепь Маркова со счетным множеством состояний $\Theta=\left\{\theta_{1}, \theta_{2}, \ldots, \theta_{k}, \ldots\right\}$ и переходной матрицей

$$
Q=\left(Q_{i j}\right)_{i, j=1}^{\infty}=\left(Q\left(\theta_{i}, \theta_{j}\right)\right)_{i, j=1}^{\infty} .
$$

С каждым значением $\theta \in \Theta$ свяжем $p$-мерный вектор $\mathbf{f}^{(\theta)}(\mathbf{s})=\left(f_{1}^{(\theta)}(\mathbf{s}), \ldots, f_{p}^{(\theta)}(\mathbf{s})\right)$, $\mathbf{s} \in \mathbf{J}^{p}$, вероятностных производящих функций $f_{i}^{(\theta)}(\mathbf{s}), i=1, \ldots, p$, соответствующих $p$-мерным распределениям вероятностей $\mathbf{F}_{i}^{(\theta)}(\{\mathbf{t}\}), \mathbf{t} \in \mathbf{N}_{0}^{p}$. Таким образом,

$$
f_{i}^{(\theta)}(\mathbf{s})=\sum_{\mathbf{t} \in \mathbf{N}_{0}^{p}} \mathbf{F}_{i}^{(\theta)}(\{\mathbf{t}\}) \mathbf{s}^{\mathbf{t}}, \quad i=1, \ldots, p .
$$

Последовательность случайных $p$-мерных векторов $\mathbf{Z}(n)=\left(Z_{1}(n), \ldots, Z_{p}(n)\right)$, $n \in \mathbf{N}_{0}$, с неотрицательными целочисленными координатами называется ветвящимся процессом Гальтона-Ватсона с $p$ типами частиц в случайной среде $\zeta$, если $\mathbf{Z}(0)$ не зависит от $\zeta$ и для всех $n \in \mathbf{N}_{0}, \mathbf{z}=\left(z_{1}, \ldots, z_{p}\right) \in \mathbf{N}_{0}^{p}$ и $k_{0}, k_{1}, \ldots \in \mathbf{N}$

$$
\mathscr{L}\left(\mathbf{Z}(n+1) \mid \mathbf{Z}(n)=\left(z_{1}, \ldots, z_{p}\right), \zeta=\left(\theta_{k_{0}}, \theta_{k_{1}}, \ldots\right)\right)=\mathscr{L}\left(\sum_{i=1}^{p} \sum_{j=1}^{z_{i}} \xi_{i}^{(j)}(n)\right),
$$

где случайные $p$-мерные векторы $\boldsymbol{\xi}_{i}^{(1)}(n), \boldsymbol{\xi}_{i}^{(2)}(n), \ldots, \boldsymbol{\xi}_{i}^{\left(z_{i}\right)}(n), i=1, \ldots, p$, имеющие целочисленные неотрицательные координаты, независимы в совокупности, и, кроме того, при фиксированном $i \in\{1, \ldots, p\}$ случайные векторы $\xi_{i}^{(1)}(n), \xi_{i}^{(2)}(n), \ldots, \xi_{i}^{\left(z_{i}\right)}(n)$ распределены согласно вероятностной мере $\mathbf{F}_{i}^{\left(\theta_{k_{n}}\right)}$.

Соотношение (2) задает ветвящийся процесс Гальтона-Ватсона в случайной среде, в котором $Z_{i}(n), i=1, \ldots, p,-$ число частиц типа $i$ в $n$-м поколении, причем частицы в этом процессе эволюционируют следующим образом. Если $\zeta_{n}=\theta \in \Theta$, то все $Z_{i}(n)$ частиц типа $i$, принадлежащие $n$-му поколению, производят потомков согласно закону распределения $\mathbf{F}_{i}^{(\theta)}$, порождаемому $p$-мерной производящей функцией $f_{i}^{(\theta)}(\mathbf{s})$, независимо от других частиц этого поколения и предыстории процесса. Таким образом, в момент времени $n+1$ потомство частицы типа $i$ из $n$-го поколения описывается случайным вектором $\xi_{i}(n)$, имеющим распределение $\mathbf{F}_{i}^{(\theta)}$. Начальное распределение вектора $\mathbf{Z}(0)$ будет указано позднее.

Для $\mathbf{z} \in \mathbf{N}_{0}^{p}, \mathbf{z} \neq \mathbf{0}$, символы $\mathbf{E}=\mathbf{E}_{\mathbf{z}}$ и $\mathbf{P}=\mathbf{P}_{\mathbf{z}}$ будут обозначать математическое ожидание и, соответственно, вероятность, относящиеся к рассматриваемому ветвящемуся процессу в случайной среде с начальным значением $\mathbf{Z}(0)=\mathbf{z}$.

Символы $\mathbb{E}_{\zeta}$ и $\mathbb{P}_{\zeta}$, играющие существенную роль в последующих рассуждениях, используются для обозначения условного математического ожидания и условной вероятности при фиксированной среде $\left(\zeta_{0}, \zeta_{1}, \zeta_{2}, \ldots\right)$. Символы $\mathscr{E} \zeta_{n}$ и $\mathscr{P}_{\zeta_{n}}$ обозначают условное математическое ожидание и условную вероятность при фиксированной векторнозначной вероятностной производящей функции $\mathbf{f}^{\left(\zeta_{n}\right)}=\mathbf{f}^{\left(\zeta_{n}\right)}(\mathbf{s})=\left(f_{1}^{\left(\zeta_{n}\right)}(\mathbf{s}), \ldots, f_{p}^{\left(\zeta_{n}\right)}(\mathbf{s})\right)^{\prime}, \mathbf{s} \in \mathbf{J}^{p}$, компонентами которой служат вероятностные производящие функции от $p$ переменных.

Всюду в дальнейшем будем предполагать, что для всех $\theta \in \Theta, i=1, \ldots, p$, у производящих функций $f_{i}^{(\theta)}(\mathbf{s})$ существуют все частные производные первого и второго порядка в точке 1. Пусть

$$
M^{(\theta)}=\left(M^{(\theta)}(i, j)\right)_{i, j=1}^{p}=\left(\frac{\partial f_{i}^{(\theta)}(\mathbf{1})}{\partial s_{j}}\right)_{i, j=1}^{p}
$$


И

$$
M_{n}=\left(M_{n}(i, j)\right)_{i, j=1}^{p}=M^{\left(\zeta_{n}\right)}=\left(\frac{\partial f_{i}^{\left(\zeta_{n}\right)}(\mathbf{1})}{\partial s_{j}}\right)_{i, j=1}^{p}
$$

- матрицы средних значений, соответствующие производящим вектор-функциям $\mathbf{f}^{(\theta)}(\mathbf{s})$ и $\mathbf{f}^{\left(\zeta_{n}\right)}(\mathbf{s})$.

Введем класс $\mathscr{C}_{b}, 0<b<1$, всех матриц $A=(A(i, j))_{i, j=1}^{p}$ с положительными элементами, таких, что

$$
b \leqslant \frac{A\left(i_{1}, j_{1}\right)}{A\left(i_{2}, j_{2}\right)} \leqslant b^{-1}, \quad 1 \leqslant i_{1}, i_{2}, j_{1}, j_{2} \leqslant p .
$$

Нас будут интересовать многотипные ветвящиеся процессы $\mathbf{Z}(n), n \in \mathbf{N}_{0}$, в случайной среде $\zeta$, для которых выполнено следующее условие.

Условие АО. Существует число $0<b<1$ такое, что для всех $\theta \in \Theta$

$$
M^{(\theta)} \in \mathscr{C}_{b} \text {. }
$$

Кроме того, будем предполагать, что пространство $\Theta$ содержит особое выделенное состояние $\theta_{1}$, у которого матрица $M^{\left(\theta_{1}\right)}$ имеет вид

$$
M^{\left(\theta_{1}\right)}=\rho\left(M^{\left(\theta_{1}\right)}\right) \mathbf{u} \otimes \mathbf{v}
$$

где

$$
\begin{gathered}
\mathbf{v}=\left(v_{1}, \ldots, v_{p}\right), \quad \mathbf{u}=\left(u_{1}, \ldots, u_{p}\right)^{\prime}, \quad v_{i}>0, \quad u_{i}>0, \quad 1 \leqslant i \leqslant p, \\
|\mathbf{u}|=1, \quad(\mathbf{v}, \mathbf{u})=1 .
\end{gathered}
$$

Ясно, что

$$
\mathbf{v}=\mathbf{v}\left(M^{\left(\theta_{1}\right)}\right), \quad \mathbf{u}=\mathbf{u}\left(M^{\left(\theta_{1}\right)}\right) .
$$

Начальным состоянием нашей среды будет именно это выделенное состояние: $\zeta_{0}=\theta_{1}$. Обозначим $\eta \geqslant 1$ момент первого возвращения марковской цепи $\zeta$ в состояние $\theta_{1}$. Пусть

$$
\rho=\rho\left(\prod_{i=0}^{\eta-1} M^{\left(\zeta_{i}\right)}\right), \quad X=\log \rho,
$$

a $\left\{X_{i}, i=1,2, \ldots\right\}$ - последовательность независимых случайных величин, имеющих то же распределение, что и величина $X$.

Положим

$$
\Sigma_{0}=0, \quad \Sigma_{n}=X_{1}+\ldots+X_{n}, \quad n \geqslant 1,
$$

и будем говорить, что случайное блуждание $\left\{\Sigma_{n}, n \geqslant 0\right\}$ порождено случайной величиной $X$. Пусть $\xi_{i}(n)-$ случайный $p$-мерный вектор, который описывает существующее в момент времени $n+1$ потомство частицы типа $i$ из $n$-го поколения.

В дальнейшем предполагается, что ветвящийся процесс $\mathbf{Z}(n), n \in \mathbf{N}_{0}$, в случайной среде $\zeta$ удовлетворяет следующим условиям. 
Условие А1. Найдется $\delta>0$ такое, что

$$
\mathbf{E} \eta^{2+\delta}<\infty
$$

Условие А2. При всех $n \geqslant 0$ выполняется соотношение

$$
\mathbf{P}\left(\min _{1 \leqslant i \leqslant p} \mathscr{P}_{\zeta_{n}}\left(\left|\boldsymbol{\xi}_{i}(n)\right|>1\right)>0\right)=1 .
$$

Условие В1. Существуют числа $b_{n}>0, n \geqslant 1$, что нормированные суммы $\Sigma_{n} / b_{n}$ сходятся по распределению к устойчивому распределению $\lambda$ с параметром $\alpha \in(0,2]$, причем $0<\lambda\left(\mathbf{R}_{+}\right)<1$.

Известно, что для случайного блуждания $\Sigma_{n}$, удовлетворяющего условию В1,

$$
b_{n}=n^{1 / \alpha} l(n),
$$

где $l(n)$ - медленно меняющаяся на бесконечности функция, и для $\Sigma_{n}$ выполняется следующее условие Спицера-Дони [27].

Условие С1. Существует число $a \in(0,1)$ такое, что при $n \rightarrow \infty$

$$
\mathbf{P}\left(\Sigma_{n}>0\right) \rightarrow a .
$$

Отметим, что для случайного блуждания $\Sigma_{n}$, удовлетворяющего условию В1,

$$
a=\lambda\left(\mathbf{R}_{+}\right)
$$

(см., например, [28]).

Для $0 \leqslant k \leqslant n$ положим

$$
\begin{aligned}
M_{k, n} & =M_{k} \cdots M_{n}, \\
\rho_{k, n} & =\rho\left(M_{k, n}\right), \\
\rho_{k} & =\rho\left(M_{k}\right) .
\end{aligned}
$$

Пусть

$$
\begin{aligned}
& S_{0}=0, \\
& S_{n}=\log \rho\left(M_{0, n-1}\right), \quad n \in \mathbf{N} .
\end{aligned}
$$

Введем случайные величины

$$
\begin{aligned}
\sigma_{n} & =\rho_{n}^{-2}\left(\max _{i, k} \sum_{j=1}^{p} \frac{\partial^{2} f_{k}^{\left(\zeta_{n}\right)}(\mathbf{1})}{\partial s_{j} \partial s_{i}}+1\right), \\
\mu & =\sum_{i=0}^{\eta-1} \sigma_{i} e^{-S_{i}} .
\end{aligned}
$$

Положим

$$
g(x)=\sum_{k=0}^{\infty} \mathbf{P}(\mu \geqslant x, \eta>k) .
$$


Условие А3. Существует

$$
\beta>\frac{1-a}{1 / \alpha+a}
$$

такое, что

$$
\lim _{x \rightarrow \infty} x^{\beta} g(x)=0
$$

Для $0 \leqslant k \leqslant n$ введем итерации производящих функций $\mathbf{f}^{\left(\zeta_{k}\right)}(\mathbf{s}), \mathbf{f}^{\left(\zeta_{k+1}\right)}(\mathbf{s}), \ldots, \mathbf{f}^{\left(\zeta_{n}\right)}(\mathbf{s})$

$$
\mathbf{f}^{k, n}(\mathbf{s})=\mathbf{f}^{\left(\zeta_{k}\right)}\left(\mathbf{f}^{\left(\zeta_{k+1}\right)}\left(\ldots \mathbf{f}^{\left(\zeta_{n}\right)}(\mathbf{s})\right)\right) .
$$

Пусть, далее,

$$
\mathbf{f}^{0, \eta-1}(\mathbf{s})=\left(f_{1}^{0, \eta-1}(\mathbf{s}), \ldots, f_{p}^{0, \eta-1}(\mathbf{s})\right)=\mathbf{f}^{\left(\zeta_{0}\right)}\left(\mathbf{f}^{\left(\zeta_{1}\right)}\left(\ldots \mathbf{f}^{\left(\zeta_{\eta-1}\right)}(\mathbf{s})\right)\right)
$$

- итерации, построенные по последовательности производящих функций $\mathbf{f}^{\left(\zeta_{0}\right)}(\mathbf{s}), \mathbf{f}^{\left(\zeta_{1}\right)}(\mathbf{s})$, $\ldots, \mathbf{f}^{\left(\zeta_{\eta-1}\right)}(\mathbf{s})$, а $\mathbf{F}_{i}^{0, \eta-1}(\{\mathbf{t}\}), \mathbf{t}=\left(t_{1}, \ldots, t_{p}\right) \in \mathbf{N}_{0}^{p},-p$-мерное распределение вероятностей на $\mathbf{N}_{0}^{p}$, соответствующее производящей функции $f_{i}^{0, \eta-1}(\mathbf{s})$ :

$$
f_{i}^{0, \eta-1}(\mathbf{s})=\sum_{\mathbf{t} \in \mathbf{N}_{0}^{p}} \mathbf{F}_{i}^{0, \eta-1}(\{\mathbf{t}\}) \mathbf{s}^{\mathbf{t}}, \quad i=1, \ldots, p .
$$

Для $d \in \mathbf{N}_{0}$ положим

$$
\begin{aligned}
& \mathbf{U}_{d}^{p}=\left\{\mathbf{t}=\left(t_{1}, \ldots, t_{p}\right) \in \mathbf{N}_{0}^{p}: t_{i}<d, i=1, \ldots, p\right\}, \\
& \mathbf{N}_{d}^{p}=\mathbf{N}_{0}^{p} \backslash \mathbf{U}_{d}^{p} .
\end{aligned}
$$

Наше следующее условие связано со случайной величиной

$$
\varkappa(d)=\frac{1}{\rho^{2}} \sum_{\mathbf{t} \in \mathbf{N}_{d}^{p}} \sum_{j, k, i=1}^{p} \mathbf{F}_{i}^{0, \eta-1}(\{\mathbf{t}\}) t_{j} t_{k} .
$$

Условие В2. Существуют числа $\varepsilon>0$ и $d \in \mathbf{N}_{0}$ такие, что

$$
\mathbf{E}\left(\log ^{+} \varkappa(d)\right)^{\alpha+\varepsilon}<\infty
$$

где величина $\alpha$ та же, что и в условии В1.

Для фиксированного начального значения $\mathbf{Z}(0)=\mathbf{z} \neq \mathbf{0}$ определим процесс $W(n)=W(n, \mathbf{z})$ соотношением

$$
W(n)=\frac{(\mathbf{Z}(n), \mathbf{u})}{\exp \left\{S_{n}\right\}}
$$

Теорема 1. Пусть выполнень условия А0, A1, А2, А3, В1 и В2. Тогда при $n \rightarrow \infty$

$$
\mathscr{L}(W(n) \mid \mathbf{Z}(n) \neq \mathbf{0} ; \mathbf{Z}(0)=\mathbf{z}) \stackrel{d}{\rightarrow} \mathscr{L}\left(W_{\mathbf{z}}\right),
$$

где случайная величина $W_{\mathbf{z}}$ положительна и конечна с вероятностью 1. 
Здесь и далее символ $\stackrel{d}{\rightarrow}$ обозначает сходимость по распределению.

Для случая процесса $Z(n), n \in \mathbf{N}_{0}$, с одним типом частиц и начальным значением $Z(0)=1$ теорема 1 принимает следующий вид.

Следствие 1. Если $p=1$ и выполняются условия $\mathrm{A} 1, \mathrm{~A} 2, \mathrm{~A} 3, \mathrm{~B} 1$ и $\mathrm{B} 2$, в которых

$$
\begin{aligned}
X & =\sum_{i=0}^{\eta-1} \log \left(\frac{d}{d s} f^{\left(\zeta_{i}\right)}(1)\right), \\
S_{i} & =\sum_{k=0}^{i-1} \log \left(\frac{d}{d s} f^{\left(\zeta_{k}\right)}(1)\right), \\
\mu & =\sum_{i=0}^{\eta-1}\left(1+\frac{d^{2}}{d s^{2}} f^{\left(\zeta_{i}\right)}(1)\left(\frac{d}{d s} f^{\left(\zeta_{i}\right)}(1)\right)^{-2}\right) e^{-S_{i}},
\end{aligned}
$$

mо при $n \rightarrow \infty$

$$
\mathscr{L}\left(\frac{Z(n)}{e^{S_{n}}} \mid Z(n)>0 ; Z(0)=1\right) \stackrel{d}{\rightarrow} \mathscr{L}(W),
$$

где случайная величина $W$ положительна и конечна с вероятностью 1.

Теорема 1 справедлива и для случайной среды, порожденной последовательностью независимых одинаково распределенных случайных величин, причем в этой ситуации от предположения счетности множества $\Theta$ можно отказаться. А именно, пусть $\widehat{\mathbf{Z}}(n)=\left(\hat{Z}_{1}(n), \ldots, \hat{Z}_{p}(n)\right)$ - процесс Гальтона-Ватсона с $p$ типами частиц, функционирующий в случайной среде $\hat{\zeta}=\left\{\hat{\zeta}_{n}, n \in \mathbf{N}_{0}\right\}$, которая задается последовательностью независимых одинаково распределенных случайных величин, определенных на вероятностном пространстве $(\Omega, \mathscr{F}, \mathscr{P})$ и принимающих значения из не обязательно счетного борелевского множества $\widehat{\Theta} \subseteq(-\infty,+\infty)$. В остальном определение процесса $\widehat{\mathbf{Z}}(n)$ совпадает с определением процесса $\mathbf{Z}(n)$ : каждому значению $\theta \in \widehat{\Theta}$ поставлен в соответствие $p$-мерный вектор $\mathbf{f}^{(\theta)}(\mathbf{s})=\left(f_{1}^{(\theta)}(\mathbf{s}), \ldots, f_{p}^{(\theta)}(\mathbf{s})\right)$ многомерных вероятностных производящих функций с матрицей средних значений $M^{(\theta)}$, и т. д. Предположим, что $\widehat{\Theta}=\widehat{\Theta}_{1} \cup \widehat{\Theta}_{2}$, где $\widehat{\Theta}_{i}, i=1,2,-$ непересекающиеся борелевские множества,

$$
\mathbf{P}\left(\theta \in \widehat{\Theta}_{1}\right)=p>0,
$$

причем для любого $\theta \in \widehat{\Theta}_{1}$ матрица $M^{(\theta)}$ имеет вид

$$
M^{(\theta)}=\rho\left(M^{(\theta)}\right) \mathbf{u} \otimes \mathbf{v}^{(\theta)},
$$

где, как и прежде,

$$
\begin{gathered}
\mathbf{v}^{(\theta)}=\left(v_{1}^{(\theta)}, \ldots, v_{p}^{(\theta)}\right), \quad \mathbf{u}=\left(u_{1}, \ldots, u_{p}\right)^{\prime}, \quad v_{i}^{(\theta)}>0, \quad u_{i}>0, \quad 1 \leqslant i \leqslant p, \\
|\mathbf{u}|=1, \quad\left(\mathbf{v}^{(\theta)}, \mathbf{u}\right)=1 .
\end{gathered}
$$

Ясно, что

$$
\mathbf{v}^{(\theta)}=\mathbf{v}\left(M^{(\theta)}\right), \quad \mathbf{u}=\mathbf{u}\left(M^{(\theta)}\right) .
$$


Отметим, что для всех матриц $M^{(\theta)}$ с параметром $\theta \in \widehat{\Theta}_{1}$ вектор $\mathbf{u}=\mathbf{u}\left(M^{(\theta)}\right)$ является одним и тем же собственным правым вектором. Пусть $\hat{\zeta}_{0}=\theta \in \widehat{\Theta}_{1}$. Условимся сохранить для момента первого возвращения среды $\hat{\zeta}$ во множество $\widehat{\Theta}_{1}$ прежнее обозначение $\eta$. Придерживаясь того же соглашения, положим

$$
\rho=\rho\left(\prod_{i=0}^{\eta-1} M^{\left(\hat{\zeta}_{i}\right)}\right), \quad X=\log \rho,
$$

и обозначим $\left\{\Sigma_{n}, n \geqslant 0\right\}$ случайное блуждание, порожденное случайной величиной $X$. Наконец, определим через соответствующие процессу $\widehat{\mathbf{Z}}(n)$ случайные величины $\rho$ и $\eta$ величину $\chi(d)$ с помощью формул (8), (9) и (10), а величину $\mu$ - с помощью формулы (6). Для фиксированного начального значения $\widehat{\mathbf{Z}}(0)=\mathbf{z} \neq \mathbf{0}$ введем процесс $\widehat{W}(n)=\widehat{W}(n, \mathbf{z})$ соотношением

$$
\widehat{W}(n)=\frac{(\widehat{\mathbf{Z}}(n), \mathbf{u})}{\exp \left\{S_{n}\right\}} .
$$

Теорема 2. Если для случайного проиесса $\widehat{\mathbf{Z}}(n)$ при соответствуюших ему случайном блуждании $\left\{\Sigma_{n}, n \geqslant 0\right\}$ и параметре $\varkappa(d)$ выполнены условия А0, А2, А3, В1 $u$ В2, то при $n \rightarrow \infty$

$$
\mathscr{L}(\widehat{W}(n, \mathbf{z}) \mid \widehat{\mathbf{Z}}(n) \neq \mathbf{0} ; \widehat{\mathbf{Z}}(0)=\mathbf{z}) \stackrel{d}{\rightarrow} \mathscr{L}\left(W_{\mathbf{z}}\right),
$$

где случайная величина $W_{\mathbf{z}}$ положительна и конечна с вероятностью 1.

\section{2. Вспомогательные результаты}

Приведем сначала несколько результатов, характеризующих произведения матриц из класса $\mathscr{C}_{b}$, упомянутого в условии А0.

Лемма 1 ([19]). Если $\left\{A_{k}, k \geqslant 1\right\}-$ последовательность положительньх матриц из класса $\mathscr{C}_{b}, 0<b<1$, то существует число $0<b^{*}<1$ такое, что при всех $n=1,2, \ldots$

$$
\prod_{k=1}^{n} A_{k} \in \mathscr{C}_{b^{*}}
$$

Лемма 2. Для элементов матрищы $A=(A(i, j))_{i, j=1}^{p} \in \mathscr{C}_{b}$ и ее правого собственного вектора $\mathbf{u}(A)=\left(u_{1}(A), \ldots, u_{p}(A)\right)$, соответствующего перронову корню $\rho(A)$, выполняются соотношения

$$
\min _{i} u_{i}(A) \geqslant \frac{b}{p}, \quad p b \leqslant \frac{\rho(A)}{A(i, j)} \leqslant p b^{-1}, \quad i, j=1, \ldots, p .
$$

Доказательство. Положим

$$
A(k)=\sum_{j=1}^{p} A(k, j), \quad k=1, \ldots, p .
$$

Так как

$$
\min _{k} A(k) \leqslant \rho(A) \leqslant \max _{k} A(k)
$$


то, в соответствии с определением класса $\mathscr{C}_{b}$,

$$
p b \leqslant \min _{k} A(k) / A(i, j) \leqslant \rho(A) / A(i, j) \leqslant \max _{k} A(k) / A(i, j) \leqslant p b^{-1} .
$$

Поскольку

$$
\sum_{j=1}^{p} u_{j}(A)=1, \quad u_{j}(A)>0,
$$

получаем, что

$$
u_{i}(A)=\sum_{j=1}^{p} \frac{A(i, j)}{\rho(A)} u_{j}(A) \geqslant b / p .
$$

Лемма доказана.

Лемма 3 ([19]). Пусть $A=(A(i, j))_{i, j=1}^{p}-$ матрица, все элементь которой положительны, а матрица $M^{\left(\theta_{1}\right)}$ имеет вид

$$
M^{\left(\theta_{1}\right)}=\rho\left(M^{\left(\theta_{1}\right)}\right) \mathbf{u} \otimes \mathbf{v}
$$

где векторы $\mathbf{u}$ u $\mathbf{v}$ удовлетворяют условиям (3) $и$ (4). Тогда

$$
\begin{aligned}
\mathbf{v}\left(A M^{\left(\theta_{1}\right)}\right) & =\mathbf{v}, \\
\mathbf{u}\left(M^{\left(\theta_{1}\right)} A\right) & =\mathbf{u}, \\
\rho\left(A M^{\left(\theta_{1}\right)}\right)=\rho\left(M^{\left(\theta_{1}\right)} A\right) & =\rho\left(M^{\left(\theta_{1}\right)}\right)(\mathbf{v}, A \mathbf{u}) .
\end{aligned}
$$

Пусть $0=\gamma_{0}<\gamma_{1}<\ldots$ - строгие убывающие лестничные моменты случайного блуждания $\Sigma_{n}$. Введем функцию

$$
\begin{array}{ll}
V(x)=\sum_{i=0}^{\infty} \mathbf{P}\left(\Sigma_{\gamma_{i}} \geqslant-x\right), & x \geqslant 0 ; \\
V(x)=0, & x<0 .
\end{array}
$$

Условие С2. Существуют числа $\varepsilon>0$ и $d \in \mathbf{N}_{0}$ такие, что

$$
\mathbf{E}\left(\log ^{+} \varkappa(d)\right)^{1 / a+\varepsilon}<\infty, \quad \mathbf{E}\left(V(X)\left(\log ^{+} \varkappa(d)\right)^{1+\varepsilon}\right)<\infty,
$$

где $a$ берется из условия (5) и $\varkappa(d)$ определено соотношением (10).

Пусть, далее,

$$
\begin{aligned}
\eta(0) & =0, \\
\eta(n+1) & =\min \left\{k>\eta(n): \zeta_{k}=\theta_{1}\right\}, \quad n \in \mathbf{N}_{0},
\end{aligned}
$$

- последовательность моментов попадания марковской цепи $\zeta$ в особое состояние $\theta_{1}$. Заметим, что набор $\eta(0), \eta(1), \eta(2), \ldots$ образует процесс восстановления, причем приращения $\eta(n+1)-\eta(n), n \in \mathbf{N}_{0}$, распределены так же, как и случайная величина $\eta=\eta(1)$ с 
математическим ожиданием $\mathbf{E} \eta \geqslant 1$. Введем вложенный ветвящийся процесс при помощи равенств

$$
\mathbf{Z}^{*}(n)=\mathbf{Z}(\eta(n)), \quad n \in \mathbf{N}_{0}
$$

Отметим, что $\mathbf{f}^{\eta(n), \eta(n+1)-1}(\mathbf{s})$ является векторнозначной производящей функцией, соответствующей закону размножения частиц $n$-го поколения процесса $\mathbf{Z}^{*}(n)$. Так как наборы $\zeta_{\eta(n)}, \zeta_{\eta(n)+1}, \ldots, \zeta_{\eta(n+1)-1}, n \in \mathbf{N}_{0}$, одинаково распределены и не зависят друг от друга, то процесс $\mathbf{Z}^{*}(n), n \in \mathbf{N}_{0}$, является ветвящимся процессом Гальтона-Ватсона, эволюционирующим в случайной среде с независимыми одинаково распределенными компонентами. Обозначим $M_{n}^{*}$ матрицу средних, соответствующую закону размножения частиц $n$-го поколения процесса $\mathbf{Z}^{*}(n)$. Ясно, что

$$
M_{n}^{*}=\prod_{i=\eta(n)}^{\eta(n+1)-1} M_{i} .
$$

Пусть

$$
X_{j}^{*}=\log \rho\left(M_{j-1}^{*}\right)
$$

и

$$
\begin{aligned}
& S_{0}^{*}=0, \\
& S_{n}^{*}=X_{1}^{*}+\ldots+X_{n}^{*}
\end{aligned}
$$

- сопровождающее случайное блуждание для процесса $\mathbf{Z}^{*}(n)=\mathbf{Z}(\eta(n)), n \in \mathbf{N}_{0}$. Очевидно, что

$$
X_{j}^{*} \stackrel{d}{=} X^{*}=\log \rho\left(M_{0, \eta-1}\right) .
$$

Определим процесс $W^{*}(n)$ соотношением

$$
W^{*}(n)=\frac{\left(\mathbf{Z}^{*}(n), \mathbf{u}\right)}{\exp \left\{S_{n}^{*}\right\}} .
$$

Лемма 4. Пусть выполнены условия А0, A1, А2, А3, В1 и В2. Тогда при $n \rightarrow \infty$

$$
\begin{aligned}
& \mathbf{P}\left(\mathbf{Z}^{*}(n) \neq \mathbf{0} \mid \mathbf{Z}^{*}(0)=\mathbf{z}\right) \sim c_{\mathbf{z}} l^{*}(n) n^{-(1-a)}, \\
& \mathscr{L}\left(W^{*}(n) \mid \mathbf{Z}^{*}(n) \neq \mathbf{0} ; \mathbf{Z}^{*}(0)=\mathbf{z}\right) \stackrel{d}{\rightarrow} \mathscr{L}\left(W_{\mathbf{z}}^{*}\right),
\end{aligned}
$$

где $c_{\mathbf{z}}>0, l^{*}(n)$-медленно меняющаяся на бесконечности функция, а случайная величина $W_{\mathbf{z}}^{*}$ положительна и конечна с вероятностью 1.

Доказательство. Ясно, что

$$
M_{n}^{*}=M^{\left(\theta_{1}\right)} \prod_{i=\eta(n)+1}^{\eta(n+1)-1} M_{i},
$$

и по лемме 3

$$
\mathbf{u}\left(M_{n}^{*}\right)=\mathbf{u}, \quad n \in \mathbf{N}_{0} .
$$


Таким образом, при любом $n \in \mathbf{N}_{0}$ вектор и является правым собственным вектором матрицы $M_{n}^{*}$, отвечающим ее перронову корню $\rho_{n}^{*}=\rho\left(M_{n}^{*}\right)$. Следовательно, в силу теоремы 2 из [16] и рассуждений, приведенных при доказательстве теоремы 1 из [16], при выполнении условий С1 и С2 верны соотношения (17) и (18). Повторяя практически дословно доказательства теорем 1 и 2 из [16], с той лишь разницей, что при доказательстве леммы 1 из [16] вместо первой части доказательства леммы 2.7 из [7] нужно использовать ее вторую часть, нетрудно показать, что совокупность условий С1 и С2 в формулировках теорем 1 и 2 из [16] может быть заменена на комплекс условий В1 и В2. Лемма доказана.

Определим случайные величины $r=r(n)$ и $m=m(n)$ соотношениями

$$
\begin{aligned}
r & =r(n)=\max \{k: \eta(k)<n\}, \\
m & =m(n)=\max \left\{k<n: \zeta_{k}=\theta_{1}\right\} .
\end{aligned}
$$

Отметим, что $S_{r}^{*}=S_{m}$. Пусть $E-$ единичная матрица размера $p \times p$ и $M_{0,-1}=E$.

Лемма 5. При $n \geqslant 1$ справедливо равенство

$$
S_{n}=S_{r(n)}^{*}+\log \rho\left(M_{m(n), n-1}\right) .
$$

Доказательство. Так как при $n \geqslant 1$

$$
M_{0, n-1}=M_{0, m(n)-1} M_{m(n), n-1},
$$

то в силу леммы 3 при $m(n) \geqslant 1$

$$
\mathbf{u}=\mathbf{u}\left(M_{0, m(n)-1}\right)=\mathbf{u}\left(M_{m(n), n-1}\right) .
$$

Следовательно, при $m(n) \geqslant 1$

$$
\rho\left(M_{0, n-1}\right)=\rho\left(M_{0, m(n)-1}\right) \rho\left(M_{m(n), n-1}\right) .
$$

Логарифмируя (24), получаем (23). Лемма доказана.

Лемма 6. Пусть выполнень условия А0, А1, А2, В1 и В2. Тогда при $n \rightarrow \infty$

$$
\mathbf{P}(\mathbf{Z}(n) \neq \mathbf{0} \mid \mathbf{Z}(0)=\mathbf{z}) \sim c_{\mathbf{z}} l(n) n^{-(1-a)},
$$

где медленно меняющаяся функичя

$$
l(n)=l^{*}(n)(\mathbf{E} \eta)^{1-a},
$$

а функиия $l^{*}(n)$ и константа $c_{\mathbf{z}}>0$ те же, что и в лемме 4. В частности,

$$
\mathbf{P}\left(\mathbf{Z}(n) \neq \mathbf{0} \mid \mathbf{Z}(0)=\mathbf{e}_{i}\right) \sim c_{i} l(n) n^{-(1-a)},
$$

где константыл $c_{i}>0, i=1, \ldots, p$.

Доказательство. При доказательстве теорем 1 и 3 из [19] было показано, что если начальное распределение среды $\zeta$ имеет вид

$$
\mathbf{P}\left(\zeta_{0}=\theta_{j}\right)=Q_{1 j}, \quad j=1,2, \ldots,
$$


(см. (1)) и справедливы условия А0, А1, В1 и В2, то

$$
\mathbf{P}\left(\mathbf{Z}(n) \neq \mathbf{0} \mid \mathbf{Z}(0)=\mathbf{e}_{i}\right) \sim \frac{1}{\mathbf{E} \eta} \mathbf{P}\left(\widetilde{\mathbf{Z}}(n) \neq \mathbf{0} \mid \widetilde{\mathbf{Z}}(0)=\mathbf{e}_{i}\right) \sim \frac{c_{i}}{\mathbf{E} \eta} \tilde{l}(n) n^{-(1-a)},
$$

где вложенный ветвящийся процесс $\widetilde{\mathbf{Z}}(n)$ задается соотношением

$$
\widetilde{\mathbf{Z}}(n)=\mathbf{Z}(\eta(n)+1), \quad n \in \mathbf{N}_{0},
$$

а $\tilde{l}(n)-$ некоторая медленно меняющаяся на бесконечности функция. Применяя к нашим процессам $\mathbf{Z}(n)$ и $\mathbf{Z}^{*}(n)$ те же рассуждения, которые проводились в доказательствах теорем 1 и 3 из [19] для процессов $\mathbf{Z}(n)$ и $\widetilde{\mathbf{Z}}(n)$, и используя лемму 4, нетрудно убедиться в справедливости (25). Лемма доказана.

Для $0 \leqslant n_{1} \leqslant n_{2}$ и $\mathbf{f}^{n_{1}, n_{2}}(\mathbf{s})=\left(f_{1}^{n_{1}, n_{2}}(\mathbf{s}), \ldots, f_{p}^{n_{1}, n_{2}}(\mathbf{s})\right)$, чтобы упростить обозначения, положим

$$
\begin{aligned}
B_{k}^{n_{1}, n_{2}}(\mathbf{s}) & =\left(B_{k}^{n_{1}, n_{2}}(\mathbf{s} ; i, j)\right)_{i, j=1}^{p}=\left(\frac{\partial^{2} f_{k}^{n_{1}, n_{2}}(\mathbf{s})}{\partial s_{i} \partial s_{j}}\right)_{i, j=1}^{p}, \\
B_{k}^{n_{1}, n_{2}} & =\left(B_{k}^{n_{1}, n_{2}}(i, j)\right)_{i, j=1}^{p}=\left(B_{k}^{n_{1}, n_{2}}(\mathbf{1} ; i, j)\right)_{i, j=1}^{p}=B_{k}^{n_{1}, n_{2}}(\mathbf{1}),
\end{aligned}
$$

и пусть $\xi_{j i}\left(n_{1}, n_{2}\right)$ - число частиц типа $i$, существующих в процессе $\mathbf{Z}(n)$ в момент времени $n_{2}$ и являющихся потомками частицы типа $j$, которая жила в $n_{1}$-м поколении этого процесса. Введем случайные величины

$$
v_{j}(m, n)=\rho_{m, n-1}^{-1} \sum_{i=1}^{p} u_{i} \xi_{j i}(m, n)-u_{j}, \quad j=1, \ldots, p .
$$

Лемма 7. Для $n \geqslant 1$ имомента $m=m(n)$ последнего до $n$ попадания ичепи $\zeta$ в спеииальное состояние справедливо неравенство

$$
\mathbb{D}_{\zeta} v_{j}(m, n) \leqslant \rho_{m, n-1}^{-2}\left\|B_{j}^{m, n-1}\right\|+u_{j} \rho_{m, n-1}^{-1}, \quad j=1, \ldots, p .
$$

Здесь и далее символ $\mathbb{D}_{\zeta} \vartheta$ обозначает дисперсию случайной величины $\vartheta$ относительно меры $\mathbb{P}_{\zeta}$.

Доказательство. Для $j=1, \ldots, p$

$$
\begin{aligned}
\mathbb{D}_{\zeta}\left[v_{j}(m, n)\right] \leqslant & \rho_{m, n-1}^{-2} \mathbb{E}_{\zeta}\left(\sum_{i=1}^{p} u_{i} \xi_{j i}(m, n)\right)^{2} \\
= & \rho_{m, n-1}^{-2}\left(\sum_{i=1}^{p} u_{i}^{2} \mathbb{E}_{\zeta}\left(\xi_{j i}(m, n)\right)^{2}+2 \mathbb{E}_{\zeta}\left(\sum_{i=1}^{p} \sum_{l=i+1}^{p} u_{i} u_{l} \xi_{j i}(m, n) \xi_{j l}(m, n)\right)\right) \\
\leqslant & \rho_{m, n-1}^{-2}\left(\sum_{i=1}^{p} u_{i} \mathbb{E}_{\zeta}\left(\xi_{j i}(m, n)\left(\xi_{j i}(m, n)-1\right)\right)+\sum_{i=1}^{p} M_{m, n-1}(j, i) u_{i}\right) \\
& +\rho_{m, n-1}^{-2}\left(2 \mathbb{E}_{\zeta}\left(\sum_{i=1}^{p} \sum_{l=i+1}^{p} \xi_{j i}(m, n) \xi_{j l}(m, n)\right)\right) \\
\leqslant & \rho_{m, n-1}^{-2}\left(\left\|B_{j}^{m, n-1}\right\|+\sum_{i=1}^{p} M_{m, n-1}(j, i) u_{i}\right)=\rho_{m, n-1}^{-2}\left\|B_{j}^{m, n-1}\right\|+u_{j} \rho_{m, n-1}^{-1} .
\end{aligned}
$$

Лемма доказана. 
Лемма 8. Для $n \geqslant 1$ имомента $m=m(n)$ последнего до п попадания цุепи $\zeta$ в специальное состояние справедливо неравенство

$$
\rho_{m, n-1}^{-2} \sum_{j=1}^{p}\left\|B_{j}^{m, n-1}\right\| \leqslant c \sum_{k=m}^{n-1} \sigma_{k} \rho_{m, k-1}^{-1}, \quad c>0
$$

где $\sigma_{k}$ те же, что и в (6).

Доказательство. Ввиду равенства

$$
B_{j}^{m, n-1}(\mathbf{s} ; i, l)=\frac{\partial^{2} f_{j}^{m, n-2}\left(\mathbf{f}^{\left(\xi_{n-1}\right)}(\mathbf{s})\right)}{\partial s_{i} \partial s_{l}},
$$

несложно видеть, что

$$
B_{j}^{m, n-1}(i, l)=\sum_{k, t=1}^{p} B_{j}^{m, n-2}(k, t) M_{n-1}(k, i) M_{n-1}(t, l)+\sum_{t=1}^{p} M_{m, n-2}(j, t) B_{t}^{n-1, n-1}(i, l)
$$

Пусть $D_{k}=\left(D_{k}(i, j)\right)_{i, j=1}^{p}-$ матрица с элементами

$$
D_{k}(i, j)=\sum_{l=1}^{p} \frac{\partial^{2} f_{i}^{\left(\zeta_{k}\right)}(\mathbf{1})}{\partial s_{l} \partial s_{j}}=\sum_{l=1}^{p} B_{i}^{k, k}(l, j) .
$$

Ясно, что

$$
D_{k}(i, j) \leqslant \hat{\sigma}_{k} \rho_{k} M_{k}(i, j),
$$

где

$$
\hat{\sigma}_{k}=\rho_{k}^{-1} \max _{i, j} \frac{D_{k}(i, j)}{M_{k}(i, j)} .
$$

Так как по лемме 2 отношение перронова корня $\rho_{k}$ матрицы $M_{k}$ к любому ее элементу $M_{k}(i, j)$ отделено от нуля и бесконечности, то

$$
\hat{\sigma}_{k} \leqslant \rho_{k} \max _{i, j} \frac{1}{M_{k}(i, j)} \rho_{k}^{-2} \max _{i, j} D_{k}(i, j) \leqslant C_{1} \sigma_{k},
$$

где $C_{1}>0$. Объединяя (28)-(32), получаем

$$
B_{j}^{m, n-1}(i, l)=\left(M_{n-1}^{\prime} B_{j}^{m, n-2} M_{n-1}\right)(i, l)+C_{1} \sigma_{n-1} \rho_{n-1} M_{m, n-1}(j, l) .
$$

Пусть $\mathscr{I}$ - матрица размера $p \times p$, все элементы которой равны 1 . Соотношение (33) дает

$$
B^{m, n-1}=\sum_{j=1}^{p} B_{j}^{m, n-1} \leqslant M_{n-1}^{\prime} B^{m, n-2} M_{n-1}+C_{1} \sigma_{n-1} \rho_{n-1} \Im M_{m, n-1} .
$$

Из оценки (34), применяя итерирование необходимое число раз и учитывая, что

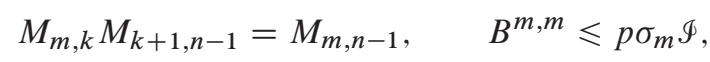


получаем

$$
\left\|B^{m, n-1}\right\| \leqslant C_{2} \sum_{k=m}^{n-1} \sigma_{k} \rho_{k}\left\|M_{k+1, n-1}^{\prime} \Phi M_{m, n-1}\right\|,
$$

где $C_{2}>0$, а $M_{k, l}=E$ при $k>l$. Положим

$$
\underline{u}=\min _{i} u_{i}, \quad \bar{u}=\max _{i} u_{i}
$$

Введем матрицу $R=(R(i, j))_{i, j=1}^{p}$, все столбцы которой одинаковы, причем $R(i, j)=u_{i}$. По лемме $3, \mathbf{u}=\mathbf{u}\left(M_{m, k}\right)$ при всех $k \geqslant m$. Применяя леммы 1,2 и учитывая, что $\|\mathscr{}\|=p^{2}$, получаем, что

$$
\begin{aligned}
\left\|M_{k+1, n-1}^{\prime} \mathscr{I} M_{m, n-1}\right\| & \leqslant \frac{1}{\underline{u}}\left\|M_{k+1, n-1}^{\prime} \Phi M_{m, n-1} R\right\|=\frac{\rho_{m, n-1}}{\underline{u}}\left\|M_{k+1, n-1}^{\prime} \Phi R\right\| \\
& =\frac{\rho_{m, n-1}}{\underline{u}}\left\|M_{k+1, n-1}^{\prime} \Phi\right\| \leqslant \frac{\rho_{m, n-1} p^{2}}{\underline{u}}\left\|M_{k+1, n-1}\right\| \\
& =\frac{\rho_{m, n-1} p^{2}}{\underline{u}}\left\|E M_{k+1, n-1}\right\| \leqslant \frac{\rho_{m, n-1} p^{3}}{\rho_{m, k} b^{*} \underline{u}}\left\|M_{m, k} M_{k+1, n-1} E\right\| \\
& \leqslant C_{3} \frac{\rho_{m, n-1}}{\rho_{m, k}}\left\|M_{m, n-1} R\right\|=C_{3} \frac{\rho_{m, n-1}^{2}}{\rho_{m, k}}\|R\|=C_{3} p \frac{\rho_{m, n-1}^{2}}{\rho_{m, k}},
\end{aligned}
$$

где

$$
C_{3}=\frac{p^{3}}{b^{*} \underline{u}^{2}}
$$

В силу лемм 1 и 2,

$$
\begin{aligned}
\rho_{m, k} & =\frac{1}{p}\left\|M_{m, k-1} M_{k} R\right\| \geqslant \frac{\rho_{k} b^{*}}{p}\left\|M_{m, k-1} \Phi R\right\| \\
& =\frac{\rho_{k} b^{*}}{p}\left\|M_{m, k-1} \Phi\right\| \geqslant \frac{\rho_{k} b^{*}}{p \bar{u}}\left\|M_{m, k-1} R\right\| \\
& =\frac{\rho_{k} \rho_{m, k-1} b^{*}}{\bar{u}} .
\end{aligned}
$$

Объединяя соотношения (34), (35), (37) и (38), приходим к (27). Лемма доказана.

Из лемм 7 и 8 вытекает следующее утверждение.

Следствие 2. Для $n \geqslant 1$ и момента $m=m(n)$ последнего до $n$ попадания ичепи $\zeta$ в специиальное состояние справедливо неравенство

$$
\sum_{j=1}^{p} \mathbb{D}_{\zeta} v_{j}(m, n) \leqslant c \sum_{k=m}^{n-1} \sigma_{k} \rho_{m, k-1}^{-1}+\rho_{m, n-1}^{-1},
$$

где константа с та же, что и в лемме 8. 


\section{3. Доказательства теорем}

Доказательство теоремы 1. Рассмотрим при $x \geqslant 0$

$$
\mathbf{P}(W(n)>x, \mathbf{Z}(n) \neq \mathbf{0})=\mathbf{P}(W(n)>x) .
$$

Пусть $\xi_{j i}^{(k)}\left(n_{1}, n_{2}\right)$ - число частиц типа $i$ в процессе $\mathbf{Z}(n)=\left(Z_{1}(n), \ldots, Z_{p}(n)\right)$ в момент времени $n_{2}$, являющихся потомками $k$-й, $k=1, \ldots, Z_{j}\left(n_{1}\right)$, частицы типа $j$, которая жила в $n_{1}$-м поколении. Используя лемму 5 , несложно видеть, что

$$
\begin{aligned}
W(n)-W^{*}(r(n)) & =\frac{(\mathbf{Z}(n), \mathbf{u})}{\exp \left\{S_{n}\right\}}-\frac{\left(\mathbf{Z}^{*}(r), \mathbf{u}\right)}{\exp \left\{S_{r}^{*}\right\}} \\
& =\frac{1}{e^{S_{r}^{*}}} \sum_{j=1}^{p}\left(\sum_{k=1}^{Z_{j}^{*}(r)}\left(\sum_{i=1}^{p} \frac{u_{i} \xi_{j i}^{(k)}(m, n)}{\rho_{m, n-1}}-u_{j}\right)\right)=\varkappa_{r, n},
\end{aligned}
$$

причем $\xi_{j i}^{(k)}(m, n) \stackrel{d}{=} \xi_{j i}(m, n)$, где случайные величины $\xi_{j i}(m, n)-$ те же, что и в (26). Отсюда получаем, что

$$
\mathbf{P}(W(n)>x)=\mathbf{P}\left(W^{*}(r)+\varkappa_{r, n}>x, \mathbf{Z}^{*}(r) \neq \mathbf{0}\right) .
$$

Пусть

$$
T=\mathbf{E} \eta
$$

Зафиксируем произвольное $\varepsilon_{0} \in(0,1 / 2)$. Положим

$$
U_{n}=U_{n}\left(\varepsilon_{0}\right)=\left[n\left(1-\varepsilon_{0}\right) / T, n\left(1+\varepsilon_{0}\right) / T\right] .
$$

Для произвольного $\varepsilon>0$ оценим

$$
\mathbf{P}\left(\left|\varkappa_{r, n}\right|>\varepsilon\right)=\mathbf{P}\left(\left|\varkappa_{r, n}\right|>\varepsilon, r \in U_{n}\right)+\mathbf{P}\left(\left|\varkappa_{r, n}\right|>\varepsilon, r \notin U_{n}\right) .
$$

Из формул (36) и (37) работы [19] следует, что

$$
\mathbf{P}\left(\left|\varkappa_{r, n}\right|>\varepsilon, r \notin U_{n}\right) \leqslant \mathbf{P}\left(\mathbf{Z}(n) \neq \mathbf{0}, r(n) \notin U_{n}\right)=o\left(n^{-\delta-1}\right), \quad n \rightarrow \infty,
$$

где величина $\delta$ та же, что и в условии A1. Покажем, что для функции

$$
y_{n}=l(n) n^{-(1-a)},
$$

где медленно меняющаяся на бесконечности функция $l(n)-$ та же, что и в лемме 6 ,

$$
\mathbf{P}\left(\left|\varkappa_{r, n}\right|>\varepsilon, r \in U_{n}\right)=o\left(y_{n}\right), \quad n \rightarrow \infty .
$$

В силу условия $\mathrm{A} 3$, найдутся числа $\varepsilon_{1} \in(0,1 / \alpha+a)$ и $\varepsilon_{2}>0$ такие, что

$$
\beta=\frac{1-a+\varepsilon_{2}}{1 / \alpha+a-\varepsilon_{1}} .
$$

Пусть

$$
\tau^{*}(n)=\min \left\{j \leqslant n: S_{j}^{*}=\min \left(S_{0}^{*}, \ldots, S_{n}^{*}\right)\right\}
$$


- самая левая точка, в которой случайное блуждание $S_{0}^{*}, \ldots, S_{n}^{*}$ на интервале $[0, n]$ достигает минимума. Для $0 \leqslant l \leqslant k$ обозначим

$$
\begin{aligned}
L_{l, k}^{*} & =\min _{0 \leqslant j \leqslant k-l}\left(S_{l+j}^{*}-S_{l}^{*}\right), \\
L_{k}^{*} & =L_{0, k}^{*} .
\end{aligned}
$$

Таким образом, $L_{k}^{*}$ - минимум случайного блуждания $S_{0}^{*}, \ldots, S_{k}^{*}$ на интервале $[0, k]$. Заметим, что

$$
\left\{\tau^{*}(k)=l\right\}=\left\{\tau^{*}(l)=l\right\} \cap\left\{L_{l, k}^{*} \geqslant 0\right\}
$$

Положим

$$
\begin{aligned}
\mathbf{f}_{*}^{k}(\mathbf{s}) & =\mathbf{f}^{\left(\zeta_{\eta(k)}\right)}\left(\mathbf{f}^{\left(\zeta_{\eta(k)+1}\right)}\left(\ldots \mathbf{f}^{\left(\zeta_{\eta(k+1)-1}\right)}(\mathbf{s})\right)\right), & & k \geqslant 0, \\
\mathbf{f}_{*}^{l, k}(\mathbf{s}) & =\mathbf{f}_{*}^{l}\left(\mathbf{f}_{*}^{l+1}\left(\ldots \mathbf{f}_{*}^{k}(\mathbf{s})\right)\right), \quad M_{l, k}^{*}=M_{l}^{*} \cdots M_{k}^{*}, & & 0 \leqslant l \leqslant k .
\end{aligned}
$$

В силу леммы 1 и условия А0, $M_{0, l-1}^{*} \in \mathscr{C}_{b^{*}}$. Применяя лемму 2 к $M_{0, l-1}^{*}$ и учитывая, чTO

$$
\rho\left(M_{0, l-1}^{*}\right)=e^{S_{l}^{*}}
$$

получаем, что при любом $k>0$

$$
\begin{aligned}
& \mathbf{P}\left(\mathbf{Z}^{*}(n) \neq \mathbf{0}, \tau^{*}(n)>k\right) \leqslant \mathbf{P}\left(\mathbf{Z}^{*}\left(\tau^{*}(n)\right) \neq \mathbf{0}, \tau^{*}(n)>k\right) \\
& =\sum_{l=k+1}^{n} \mathbf{P}\left(\mathbf{Z}^{*}(l) \neq \mathbf{0}, \tau^{*}(l)=l, L_{l, n}^{*} \geqslant 0\right) \\
& =\sum_{i=k+1}^{n} \mathbf{P}\left(\mathbf{Z}^{*}(l) \neq \mathbf{0}, \tau^{*}(l)=l\right) \mathbf{P}\left(L_{n-l}^{*} \geqslant 0\right) \\
& \leqslant|\mathbf{z}| \sum_{i=k+1}^{n} \mathbf{E}\left(\left|\mathbf{1}-\mathbf{f}_{*}^{0, l-1}(\mathbf{0})\right| ; \tau^{*}(l)=l\right) \mathbf{P}\left(L_{n-l}^{*} \geqslant 0\right) \\
& \leqslant|\mathbf{z}| \sum_{i=k+1}^{n} \mathbf{E}\left(\mathbf{1}^{\prime} M_{0, l-1}^{*} \mathbf{1} ; \tau^{*}(l)=l\right) \mathbf{P}\left(L_{n-l}^{*} \geqslant 0\right) \\
& \leqslant \frac{p|\mathbf{z}|}{b^{*}} \sum_{i=k+1}^{n} \mathbf{E}\left(e^{S_{l}^{*}} ; \tau^{*}(l)=l\right) \mathbf{P}\left(L_{n-l}^{*} \geqslant 0\right),
\end{aligned}
$$

где $\mathbf{z}=\mathbf{Z}(\mathbf{0})=\mathbf{Z}^{*}(\mathbf{0})$ и $b^{*}$ взяты из (14). Так как в силу условия В1 сопровождающее случайное блуждание процесса $\mathbf{Z}^{*}(n)$ удовлетворяет условию $\mathrm{C} 1$, то из (44) следует (см. формулу (4.3) статьи [7]), что

$$
\lim _{k \rightarrow \infty} \limsup _{n \rightarrow \infty} y_{n}^{-1} \mathbf{P}\left(\mathbf{Z}^{*}(n) \neq \mathbf{0}, \tau^{*}(n)>k\right)=0 .
$$

Для $M=\left[n^{\varepsilon_{1} / 4}\right]$ (здесь и далее $[x]$ обозначает целую часть числа $x$ ) запишем представление

$$
\begin{aligned}
\mathbf{P}\left(\left|\varkappa_{r, n}\right|>\varepsilon, r \in U_{n}\right)= & \mathbf{P}\left(\left|\varkappa_{r, n}\right|>\varepsilon, \tau^{*}(r)>M, r \in U_{n}\right) \\
& \quad+\mathbf{P}\left(\left|\varkappa_{r, n}\right|>\varepsilon, \tau^{*}(r) \leqslant M, r \in U_{n}\right) \\
= & \mathscr{H}(n, M)+\mathscr{A}(n, M) .
\end{aligned}
$$


Несложно видеть, что

$$
\begin{aligned}
\mathscr{H}(n, M) & \leqslant \mathbf{P}\left(\mathbf{Z}^{*}(r) \neq \mathbf{0}, \tau^{*}(r)>M, r \in U_{n}\right) \\
& \leqslant \mathbf{P}\left(\mathbf{Z}^{*}\left(\left[n\left(1-\varepsilon_{0}\right) / T\right]\right) \neq \mathbf{0}, \tau^{*}\left(\left[n\left(1+\varepsilon_{0}\right) / T\right]\right)>M\right) \\
& \leqslant \mathscr{H}_{1}(n, M)+\mathscr{H}_{2}(n),
\end{aligned}
$$

где

$$
\begin{aligned}
\mathscr{H}_{1}(n, M) & =\mathbf{P}\left(\mathbf{Z}^{*}\left(\left[n\left(1+\varepsilon_{0}\right) / T\right]\right) \neq \mathbf{0}, \tau^{*}\left(\left[n\left(1+\varepsilon_{0}\right) / T\right]\right)>M\right), \\
\mathscr{H}_{2}(n) & =\mathbf{P}\left(\mathbf{Z}^{*}\left(\left[n\left(1-\varepsilon_{0}\right) / T\right]\right) \neq \mathbf{0}, \mathbf{Z}^{*}\left(\left[n\left(1+\varepsilon_{0}\right) / T\right]\right)=\mathbf{0}\right) .
\end{aligned}
$$

В силу (45),

$$
\limsup _{n \rightarrow \infty} y_{n}^{-1} \mathscr{H}_{1}(n, M)=0
$$

Так как

$$
\mathscr{H}_{2}(n)=\mathbf{P}\left(\mathbf{Z}^{*}\left(\left[n\left(1-\varepsilon_{0}\right) / T\right]\right) \neq \mathbf{0}\right)-\mathbf{P}\left(\mathbf{Z}^{*}\left(\left[n\left(1+\varepsilon_{0}\right) / T\right]\right) \neq \mathbf{0}\right),
$$

учитывая, что $\varepsilon_{0} \in(0,1 / 2)$, в силу (17) получаем

$$
\begin{aligned}
\lim _{n \rightarrow \infty} \frac{\mathscr{H}_{2}(n)}{y_{n}} & =c_{\mathbf{Z}}\left(\left(1-\varepsilon_{0}\right)^{-(1-a)}-\left(1+\varepsilon_{0}\right)^{-(1-a)}\right) \\
& \leqslant c_{\mathbf{z}}\left(\left(\frac{1+\varepsilon_{0}}{1-\varepsilon_{0}}\right)^{1-a}-1\right) \leqslant c_{\mathbf{z}}\left(\frac{1+\varepsilon_{0}}{1-\varepsilon_{0}}-1\right) \leqslant 4 c_{\mathbf{z}} \varepsilon_{0} .
\end{aligned}
$$

Оценим теперь величину $\mathscr{A}(n, M)$. Для $N=\left[n^{\varepsilon_{1} / 2}\right]$ представим $\mathscr{A}(n, M)$ в виде

$$
\mathscr{A}(n, M)=\mathscr{A}_{1}(n, M, N)+\mathscr{A}_{2}(n, M, N),
$$

где

$$
\begin{aligned}
& \mathscr{A}_{1}(n, M, N)=\sum_{l=0}^{M} \mathbf{P}\left(\left|\varkappa_{r, n}\right|>\varepsilon, Z^{*}(r) \neq \mathbf{0}, e^{-S_{l}^{*}}>N, \tau^{*}(r)=l, r \in U_{n}\right), \\
& \mathscr{A}_{2}(n, M, N)=\sum_{l=0}^{M} \mathbf{P}\left(\left|\varkappa_{r, n}\right|>\varepsilon, Z^{*}(r) \neq \mathbf{0}, e^{\left.-S_{l}^{*} \leqslant N, \tau^{*}(r)=l, r \in U_{n}\right) .}\right.
\end{aligned}
$$

Ясно, что

$$
\begin{aligned}
\mathscr{A}_{1}(n, M, N) & \leqslant \sum_{l=0}^{M} \mathbf{P}\left(\mathbf{Z}^{*}(r) \neq \mathbf{0}, e^{-S_{l}^{*}}>N, \tau^{*}(r)=l, r \in U_{n}\right) \\
& =\mathbf{E}\left(\sum_{l=0}^{M} \mathbb{P}_{\zeta}\left(\mathbf{Z}^{*}(r) \neq \mathbf{0}, e^{-S_{l}^{*}}>N, \tau^{*}(r)=l, r \in U_{n}\right)\right) \\
& \leqslant|\mathbf{z}| \mathbf{E}\left(\sum_{l=0}^{M}\left|\mathbf{1}-\mathbf{f}_{*}^{0, r-1}(\mathbf{0})\right| I\left\{e^{-S_{l}^{*}}>N, \tau^{*}(r)=l, r \in U_{n}\right\}\right) \\
& \leqslant|\mathbf{z}| \mathbf{E}\left(\sum _ { l = 0 } ^ { M } 1 M _ { 0 , l - 1 } ^ { * } ( \mathbf { 1 } - \mathbf { f } _ { * } ^ { l , r - 1 } ( \mathbf { 0 } ) ) ^ { \prime } I \left\{e^{\left.\left.-S_{l}^{*}>N, \tau^{*}(r)=l, r \in U_{n}\right\}\right),}\right.\right.
\end{aligned}
$$


где $M_{0,-1}^{*}=E$ и $I\{\mathscr{b}\}$ - индикатор события $\mathscr{C}$. Из теоремы 1 и формул (45) и (46) в [16] следует, что при $n \rightarrow \infty$

$$
\begin{aligned}
\mathbf{P}\left(\mathbf{Z}^{*}(n) \neq \mathbf{0}, L_{n}^{*} \geqslant 0 \mid \mathbf{Z}^{*}(0)=\mathbf{e}_{i}\right) & =\mathbf{E}\left(\mathbb{P}_{\zeta}\left(\mathbf{Z}^{*}(n) \neq \mathbf{0}, L_{n}^{*} \geqslant 0 \mid \mathbf{Z}^{*}(0)=\mathbf{e}_{i}\right)\right) \\
& =\mathbf{E}\left(\left(\mathbf{e}_{i},\left(\mathbf{1}-\mathbf{f}_{*}^{0, n-1}(\mathbf{0})\right)^{\prime}\right) I\left\{L_{n}^{*} \geqslant 0\right\}\right) \\
& \sim \mathbf{P}\left(\mathbf{Z}^{*}(n) \neq \mathbf{0} \mid \mathbf{Z}^{*}(0)=\mathbf{e}_{i}\right) .
\end{aligned}
$$

Из (54), применяя сначала леммы 1, 2 и 4, а затем соотношения (43) и (55), получаем, что для некоторого $C>0$ и $b^{*}$ из (14)

$$
\begin{aligned}
& \mathscr{A}_{1}(n, M, N) \leqslant \frac{p|\mathbf{z}|}{b^{*}} \mathbf{E}\left(\sum_{l=0}^{M} e^{S_{l}^{*}}\left|\mathbf{1}-\mathbf{f}_{*}^{l, r-1}(\mathbf{0})\right| I\left\{e^{-S_{l}^{*}}>N, \tau^{*}(r)=l, r \in U_{n}\right\}\right) \\
& \leqslant \frac{p|\mathbf{z}|}{N b^{*}} \mathbf{E}\left(\sum_{l=0}^{M}\left|\mathbf{1}-\mathbf{f}_{*}^{l, r-1}(\mathbf{0})\right| I\left\{\tau^{*}(r)=l, r \in U_{n}\right\}\right) \\
& =\frac{p|\mathbf{z}|}{N b^{*}} \sum_{l=0}^{M} \mathbf{P}\left(\tau^{*}(l)=l\right) \mathbf{E}\left(\left|\mathbf{1}-\mathbf{f}_{*}^{l, r-1}(\mathbf{0})\right| ; L_{l, r-1}^{*} \geqslant 0, r \in U_{n}\right) \\
& \leqslant \frac{(M+1) p|\mathbf{z}|}{N b^{*}} \mathbf{E}\left(\left|\mathbf{1}-\mathbf{f}_{*}^{l, r-1}(\mathbf{0})\right| ; L_{l, r-1}^{*} \geqslant 0, r \in U_{n}\right) \\
& \leqslant C \frac{(M+1)|\mathbf{z}|}{N} y_{n}\left(1-\varepsilon_{0}\right)^{-(1-a)}=o\left(y_{n}\right), \quad n \rightarrow \infty \text {. }
\end{aligned}
$$

Пусть

$$
m^{+}=m^{+}(n)=\min \left\{k \geqslant n: \zeta_{k}=\theta_{1}\right\}
$$

- следующий после $m(n)$ момент попадания цепи $\zeta$ в особое состояние. Для случайных величин $m=m(n)$ и $m^{+}=m^{+}(n)$ положим

$$
\begin{gathered}
\Xi=\Xi(n)=\sum_{k=m}^{n} \sigma_{k} \rho_{m, k-1}^{-1}, \\
\Xi^{(1)}=\Xi^{(1)}(n)=\sum_{k=m}^{m^{+}} \sigma_{k} \rho_{m, k-1}^{-1} .
\end{gathered}
$$

При $x_{n}=n^{1 / \alpha+a-\varepsilon_{1}}$, где $\varepsilon_{1}$ взят из (42), представим величину $\mathscr{A}_{2}(n, M, N)$ (см. (53)) в виде

$$
\mathscr{A}_{2}(n, M, N)=\mathscr{A}_{2}^{(1)}(n, M, N)+\mathscr{A}_{2}^{(2)}(n, M, N),
$$

где

$$
\begin{aligned}
& \mathscr{A}_{2}^{(1)}(n, M, N)=\sum_{l=0}^{M} \mathbf{P}\left(\left|\varkappa_{r, n}\right|>\varepsilon, e^{-S_{l}^{*}} \leqslant N, \Xi>x_{n}, \tau^{*}(r)=l, r \in U_{n}\right), \\
& \mathscr{A}_{2}^{(2)}(n, M, N)=\sum_{l=0}^{M} \mathbf{P}\left(\left|\varkappa_{r, n}\right|>\varepsilon, e^{-S_{l}^{*}} \leqslant N, \quad \Xi \leqslant x_{n}, \tau^{*}(r)=l, r \in U_{n}\right) .
\end{aligned}
$$


Положим

$$
\eta_{n}^{-}=n-S_{m(n)}, \quad G(y)=\mathbf{P}(\eta \leqslant y) .
$$

Пусть

$$
H(k)=\sum_{l=1}^{\infty} G^{l *}(k), \quad h(k+1)=H(k+1)-H(k), \quad k \in \mathbf{N}_{0},
$$

где $G^{l *}(y)-l$-кратная свертка функции распределения $G(y)$. Положим $t=\left[\left(1-\varepsilon_{0}\right) / T\right]$. Используя очевидные неравенства

$$
\Xi^{(1)} \geqslant \Xi, \quad m(n) \geqslant r(n),
$$

заключаем, что

$$
\begin{aligned}
\mathscr{A}_{2}^{(1)}(n, M, N) & \leqslant \mathbf{P}\left(\Xi>x_{n}, r \in U_{n}\right) \leqslant \mathbf{P}\left(\Xi^{(1)}>x_{n}, m(n) \geqslant n t\right) \\
& \leqslant \sum_{k=1}^{n(1-t)} \mathbf{P}\left(\Xi^{(1)}>x_{n}, \eta_{n}^{-}=k\right) \\
& =\sum_{k=1}^{n(1-t)} h(n-k) \mathbf{P}\left(\mu>x_{n}, \eta \geqslant k\right) ;
\end{aligned}
$$

отсюда в силу теоремы восстановления (см., например, [30], гл. 11, §1) получаем, что при достаточно больших $n$

$$
\mathscr{A}_{2}^{(1)}(n, M, N) \leqslant \frac{2}{T} \sum_{k=0}^{\infty} \mathbf{P}\left(\mu>x_{n}, \eta>k\right) .
$$

Из условия А3 и оценки (59) следует, что

$$
\mathscr{A}_{2}^{(1)}(n, M, N)=o\left(y_{n}\right), \quad n \rightarrow \infty .
$$

Оценим теперь $\mathscr{A}_{2}^{(2)}(n, M, N)$. Очевидно, что

$$
\mathscr{A}_{2}^{(2)}(n, M, N)=\mathbf{E}\left(\sum_{l=0}^{M} \mathbb{P}_{\zeta}\left(\left|\varkappa_{r, n}\right|>\varepsilon, e^{-S_{l}^{*}} \leqslant N, \quad \Xi \leqslant x_{n}, \tau^{*}(r)=l, r \in U_{n}\right)\right) .
$$

Пусть

$$
v_{j}^{(k)}\left(n_{1}, n_{2}\right)=\sum_{i=1}^{p} \frac{u_{i} \xi_{j i}^{(k)}\left(n_{1}, n_{2}\right)}{\rho_{n_{1}, n_{2}-1}}-u_{j}, \quad k=1, \ldots, Z_{j}\left(n_{1}\right)
$$

Тогда

$$
\varkappa_{r, n}=\frac{1}{e^{S_{r}^{*}}} \sum_{j=1}^{p}\left(\sum_{k=1}^{Z_{j}^{*}(r)} v_{j}^{(k)}(m, n)\right)=\frac{1}{e^{S_{r}^{*}}} \sum_{j=1}^{p}\left(\sum_{k=1}^{Z_{j}(m)} v_{j}^{(k)}(m, n)\right) .
$$


Отметим, что $v_{j}^{(k)}(m, n) \stackrel{d}{=} v_{j}(m, n)$, где случайные величины $v_{j}(m, n)$ те же, что и в соотношении (26). Согласно неравенству Чебышева,

$$
\mathbb{P}_{\zeta}\left(\left|\mathcal{\varkappa}_{r, n}\right|>\varepsilon, \tau^{*}(r)=l, r \in U_{n}\right) \leqslant \frac{1}{\varepsilon^{2} e^{2 S_{r}^{*}}} \mathbb{D}_{\zeta}\left(\sum_{j=1}^{p}\left(\sum_{k=1}^{Z_{j}(m)} v_{j}^{(k)}(m, n)\right)\right) .
$$

Так как при фиксированной среде $\zeta$ случайные величины $v_{j}^{(k)}(m, n), j=1, \ldots, p$, $k=1, \ldots, Z_{j}(m)$, независимы и $\mathbf{Z}(0)=\mathbf{z}$, то

$$
\begin{aligned}
\mathbb{D}_{\zeta}\left(\sum_{j=1}^{p}\left(\sum_{k=1}^{Z_{j}(m)} v_{j}^{(k)}(m, n)\right)\right) & \leqslant|\mathbf{z}| \sum_{j=1}^{p} \sum_{i=1}^{p} M_{0, m-1}(i, j) \mathbb{D}_{\zeta} v_{j}(m, n) \\
& \leqslant \frac{|\mathbf{z}|}{\min _{i} u_{i}} \sum_{k=1}^{p} \mathbb{D}_{\zeta} v_{k}(m, n) \sum_{j=1}^{p} \sum_{i=1}^{p} M_{0, m-1}(i, j) u_{j} \\
& =\frac{|\mathbf{z}|}{\min _{i} u_{i}} \exp \left\{S_{r}^{*}\right\} \sum_{k=1}^{p} \mathbb{D}_{\zeta} v_{k}(m, n) .
\end{aligned}
$$

В силу следствия 2,

$$
\sum_{k=1}^{p} \mathbb{D}_{\zeta} v_{k}(m, n) \leqslant c \Xi
$$

где $\Xi$ то же, что и в соотношении (57), а с взято из следствия 2. Из (61)-(64) получаем, что

$$
\begin{aligned}
\mathscr{A}_{2}^{(2)}(n, M, N) & \leqslant \sum_{l=0}^{M} \mathbf{E}\left(\frac{e^{-S_{l}^{*}}}{\varepsilon^{2} e^{S_{r}^{*}-S_{l}^{*}}} \Xi ; e^{-S_{l}^{*}} \leqslant N, \Xi \leqslant x_{n}, \tau^{*}(r)=l, r \in U_{n}\right) \\
& \leqslant \frac{C_{0} N x_{n}}{\varepsilon^{2}} \sum_{l=0}^{M} \mathbf{E}\left(e^{-\left(S_{r}^{*}-S_{l}^{*}\right)} ; \tau^{*}(r)=l, r \in U_{n}\right),
\end{aligned}
$$

где $C_{0}>0$. В силу утверждения 2.1 работы [8],

$$
\mathbf{E}\left(e^{-S_{k}^{*}}, L_{k}^{*} \geqslant 0\right) \sim C k^{-1-1 / \alpha} l^{-1}(k), \quad k \rightarrow \infty .
$$

Соотношения (65) и (66) дают

$$
\mathscr{A}_{2}^{(2)}(n, M, N) \leqslant \frac{C_{1} N(M+1) x_{n}}{\varepsilon^{2}} n^{-1-1 / \alpha} l^{-1}(n)=o\left(y_{n}\right), \quad n \rightarrow \infty,
$$

где $C_{1}>0$. Объединяя (46)-(52), (56), (58), (60) и (67), в силу произвольности $\varepsilon_{0}$ приходим к (41). Из (40) и (41), пользуясь леммой 4, получаем (7). Теорема доказана.

Доказательство теоремы 2. Для ветвящегося процесса $\widehat{\mathbf{Z}}(n)$ положим $\hat{\eta}(0)=0$ и обозначим $\hat{\eta}(1)$ момент первого возвращения среды $\hat{\zeta}_{n}$ во множество $\Theta_{1}$. Пусть

$$
\hat{\eta}(n+1)=\min \left\{k>\hat{\eta}(n): \hat{\zeta}_{k} \in \Theta_{1}\right\}, \quad n \in \mathbf{N}_{0} .
$$


Отметим, что в условиях теоремы 2 момент $\eta=\hat{\eta}(1)$ первого возвращения среды $\hat{\zeta}_{n}$ во множество $\Theta_{1}$ удовлетворяет условию Крамера, и, следовательно, условию А1. Теперь остается лишь применить схему доказательства теоремы 1, проинтерпретировав встречающиеся в ней величины $\eta(k)$ в соответствии с соотношением (68) как $\hat{\eta}(k)$. Теорема доказана.

Автор выражает благодарность рецензенту, чьи замечания способствовали улучшению работы.

\section{Список литературы}

1. Smith W. L., Wilkinson W., On branching processes in random environment. Ann. Math. Statist. (1969) 40, №3, 814-827.

2. Athreya K. B., Karlin S., On branching processes with random environments. I, II. Ann. Math. Statist. (1971) 42, №5; 6, 1499-1520; 1843-1858.

3. Tanny D., On multitype branching processes in a random environment. Adv. Appl. Probab. (1981) 13, №3, 464-497.

4. Weissener E. W., Multitype branching processes in random environments. J. Appl. Probab. (1971) 8, №1, 17-31.

5. Kaplan N., Some results about multidimentional branching processes with random environments. Ann. Probab. (1974) 2, №3, 441-455.

6. Афанасьев В. И., Предельная теорема для критического ветвящегося процесса в случайной среде. Дискретная математика (1993) 5, №1, 45-58.

7. Afanasyev V. I., Geiger J., Kersting G., Vatutin V. A., Criticality for branching processes in random environment. Ann. Probab. (2005) 33, №2, 645-673.

8. Afanasyev V. I., Böinghoff K., Kersting G., Vatutin V. A., Limit theorems for weakly subcritical branching processes in random environment. J. Theoretical Probab. (2012) 25, №3, 703-732.

9. Geiger J., Kersting G., The survival probability of a critical branching process in random environment. Теория вероятностей и ее применения (2000) 45, №3, 607-615.

10. Ватутин В. А., Дьяконова Е. Е., Ветвящиеся процессы Гальтона-Ватсона в случайной среде. I: предельные теоремы. Теория вероятностей и ее применения (2003) 48, №2, 274-300.

11. Ватутин В. А., Дьяконова Е. Е., Ветвящиеся процессы Гальтона-Ватсона в случайной среде. II: конечномерные распределения. Теория вероятностей и ее применения (2004) 49, №2, 231-268.

12. Ватутин В. А., Дьяконова Е. Е., Ветвящиеся процессы в случайной среде и бутылочные горлышки в эволюции популяций. Теория вероятностей и ее применения (2006) 51, №1, 22-46.

13. Ватутин В. А., Вахтель В. И., Внезапное вырождение критического ветвящегося процесса в случайной среде. Теория вероятностей и ее применения (2009) 54, №3, 417-438.

14. Ватутин В. А., Системы поллинга и многотипные ветвящиеся процессы в случайной среде. Теория вероятностей и ее применения (2010) 55, №4, 644-679.

15. Ватутин В. А., Многотипные ветвящиеся процессы с иммиграцией, эволюционирующие в случайной среде. Матем. тр. (2011) 14, №1, 3-49.

16. Ватутин В. А., Дьяконова Е. Е., Асимптотические свойства многотипных критических ветвящихся процессов, эволюционирующих в случайной среде. Дискретная математика (2010) 22, №2, 22-40.

17. Дьяконова Е. Е., Об асимптотике вероятности невырождения многомерного ветвящегося процесса в случайной среде. Дискретная математика (1999) 11, №1, 113-128. 
18. Дьяконова Е. Е., Критические многотипные ветвящиеся процессы в случайной среде. Дискретная математика (2007) 19, №4, 23-41.

19. Дьяконова Е. Е., Многотипные ветвящиеся процессы Гальтона-Ватсона в марковской случайной среде. Теория вероятностей и ее применения (2011) 55, №3, 592-601.

20. Dyakonova E. E., Geiger J., Vatutin V. A., On the survival probability and a functional limit theorem for branching processes in random environment. Markov Process. Related Fields (2004) 10, №2, 289-306.

21. D’Souza J. C., Hambly B. M., On the survival probability of a branching process in a random environment. Adv. Appl Probab. (1997) 29, №1, 38-55.

22. Roitershtein A., A note on multitype branching processes with immigration in a random environment. Ann. Probab. (2007) 35, №4, 1573-1592.

23. Козлов М. В., Об асимптотике вероятности невырождения критических ветвящихся процессов в случайной среде. Теория вероятностей и ее применения (1976) 21, №4, 813-825.

24. Kesten H., Spitzer F., Convergence in distribution of products of random matrices. Z. Wahrscheinlichkeitstheor. Verw. Geb. (1984) 67, №4, 363-386.

25. Петров В. В., Суммы независимых случайных величин. Наука, Москва, 1972.

26. Athreya K. B., Ney P. E., Branching processes. Springer,, Berlin, 1972.

27. Doney R. A., Spitzer's condition and ladder variables in random walks. Theory Probab. Related Fields (1995) 101, 577-580.

28. Золотарев В. М., Одномерные устойчивые распределения. Наука, Москва, 1983.

29. Севастьянов Б. А., Ветвящиеся процессы. Наука, Москва, 1971.

30. Феллер В., Введение в теорию вероятностей и ее приложения, 2. Мир, Москва, 1984.

Статья поступила 23.01.2012. 